\title{
Ultrasound-assisted catheter placement in CT-guided HDR brachytherapy for the local ablation of abdominal malignancies: Initial experience
}

\section{Ultraschallassistierte Katheteranlage bei der CT-geführten HDR-Brachytherapie zur lokalen Ablation abdomineller Malignome: erste Erfahrungen.}

Authors

Robert Damm¹, Shahen El-Sanosy ${ }^{1}$, Jazan Omari ${ }^{1}$, Romy Damm¹, Peter Hass ${ }^{2}$, Maciej Pech ${ }^{1}$, Maciej Powerski ${ }^{1}$

Affiliations

1 Department of Radiology and Nuclear Medicine, University of Magdeburg, Germany

2 Department of Radiotherapy, University of Magdeburg, Germany

Key words

brachytherapy, interventional procedures, ultrasound computed tomography (US/CT)

received 20.02.2018

accepted 14.05.2018

Bibliography

DOI https://doi.org/10.1055/a-0636-4055

Published online: 11.10.2018

Fortschr Röntgenstr 2019; 191: 48-53

(c) Georg Thieme Verlag KG, Stuttgart · New York

ISSN 1438-9029

Correspondence

Dr. Robert Damm

Klinik für Radiologie und Nuklearmedizin,

Universitätsklinikum Magdeburg A.ö.R.,

Leipziger Str. 44, 39120 Magdeburg, Germany

Tel.: ++49/3 91/6713030

Fax: ++49/3 91/6713029

robert.damm@med.ovgu.de

\section{ABSTRACT}

Purpose To evaluate the safety and feasibility of sonographically-assisted catheter placement in interstitial high-dose-rate brachytherapy of abdominal malignancies.

Materials and Methods In an initial cohort of 12 patients and 16 abdominal tumors (colorectal liver metastases $\mathrm{n}=9$; renal cell cancer $n=3$; hepatocellular carcinoma $n=2$; cholangiocellular carcinoma $n=2$ ), initial puncture and catheter placement for CT-guided brachytherapy were performed under sonographic assistance when possible. The interventional procedure was prospectively recorded and in-patient data were collected. All data underwent descriptive statistics and comparative analysis by the Mann-Whitney test.
Results In 12 out of 16 lesions (diameter $1.5-12.9 \mathrm{~cm}$ ), initial puncture was successfully achieved under ultrasound guidance without utilization of CT fluoroscopy, yielding a significantly shorter mean total fluoroscopy time (14.5 vs. $105.5 \mathrm{~s}$; $\mathrm{p}=0.006)$. In 8 lesions visibility was rated better in ultrasound than in CT fluoroscopy $(p=0.2)$. No major or minor complications occurred within 30 days after treatment.

Conclusion Ultrasound-assisted catheter placement during interstitial CT-guided brachytherapy of abdominal tumors could improve catheter positioning and reduce radiation exposure for medical staff.

Key points Ultrasound-assisted catheter placement in CT-guided brachytherapy is safe and feasible. Ultrasound puncture may improve catheter positioning. Reduced CT fluoroscopy time can significantly help to minimize radiation exposure for medical staff.

\section{Citation Format}

- Damm R, El-Sanosy S, Omari J et al. Ultrasound-assisted catheter placement in CT-guided HDR brachytherapy for the local ablation of abdominal malignancies: Initial experience. Fortschr Röntgenstr 2019; 191: 48-53

\section{ZUSAMMENFASSUNG}

Ziel Evaluierung der Sicherheit und Machbarkeit der sonografisch assistierten Katheter-Anlage bei der interstitiellen Highdose-rate-Brachytherapie abdomineller Malignome.

Material und Methoden In einer ersten Kohorte von $12 \mathrm{~Pa}$ tienten mit 16 abdominellen Tumoren (kolorektale Lebermetastasen $n=9$; Nierenzellkarzinom $n=3$; hepatozelluläres Karzinom $n=2$; cholangiozelluläres Karzinom $n=2$ ) erfolgte die initiale Punktion und Katheter-Anlage bei der CT-gestützten Brachytherapie soweit möglich unter sonografischer Führung. Die Durchführung des Eingriffs wurde prospektiv erfasst und der klinische Verlauf der Patienten dokumentiert. Die erhobenen Daten wurden deskriptiv ausgewertet und mit dem Mann-Whitney-U-Test analysiert.

Ergebnisse Bei 12 von 16 Läsionen (Diameter 1,5-12,9cm) konnte die initiale Punktion zur Katheter-Platzierung sonografisch erfolgreich ohne Zuhilfenahme der CT-Fluoroskopie vor- 
genommen werden, wodurch sich die mittlere Fluoroskopiezeit des gesamten Eingriffs signifikant verkürzen ließ (14,5 vs. $105,5 s ; p=0,006)$. Bei 8 Läsionen wurde die Sichtbarkeit im Ultraschall insgesamt besser bewertet als in der CT-Fluoroskopie $(p=0,2)$. Es traten keine Minor- oder Majorkomplikationen innerhalb von 30 Tagen auf.
Schlussfolgerung Die ultraschallassistierte Katheter-Anlage könnte bei der interstitiellen, CT-gestützten Brachytherapie abdomineller Tumore sowohl zur verbesserten Katheter-Positionierung als auch zur Reduzierung der Strahlenexposition des medizinischen Personals beitragen.

\section{Introduction}

CT-guided interstitial brachytherapy (HDR-BT) is a catheter-based procedure that, among other things, allows local ablation of thoracic and abdominal malignancies.

The irradiation catheter is usually positioned percutaneously using CT fluoroscopy.

Unlike thermal ablation procedures, such as radiofrequency ablation (RFA) and microwave ablation (MWA), this treatment method has no technical limitation regarding tumor size and proximity to heat-vulnerable structures [1, 2]. Furthermore, interstitial irradiation is not subject to any thermal cooling effects by adjacent vessels and has no influence on respiratory excursion due to the fixed placement of the irradiation catheter in the tumor in relation to stereotaxis (SBRT) [3, 4].

Multiple catheters need to be introduced for multiple and larger lesions resulting in a complex procedure typically associated with higher radiation exposure for the radiologist [5, 6]. Furthermore, the accessibility, especially of smaller lesions, under CT fluoroscopy is often difficult and requires experience to reach the target lesion in a dose-saving manner [7, 8].

In thermal ablation procedures, such as radiofrequency ablation, in addition to computer tomography, ultrasound in image guidance has become established and thus represents an alternative to ionizing radiation [9].

In this study we report initial experiences with sonographicallyassisted catheter positioning in the interstitial brachytherapy of hepatic and renal malignancies as a supplement to CT fluoroscopy.

\section{Materials and Methods}

\section{Patient Cohort}

Since July 2017 patients have been recruited and included in a prospective feasibility study. The inclusion criteria include patients with planned, interstitial brachytherapy for the ablation of tumors in sonographically clearly visible organs such as the liver and kidneys. The study was reviewed and approved by the local ethics committee. Prior to the procedure all patients provided their informed consent to study-specific activities and approved the further processing of their clinical and radiographic data for study purposes in accordance with data protection guidelines.

Twelve patients were included ( 8 men, 4 women, mean age 70 years) with a total of 16 tumors to be treated (colorectal liver metastases $n=9$, renal cell carcinomas $n=3$, hepatocellular carcinomas $n=2$ and cholangiocarcinomas $n=2$ ).
Three patients had prior liver surgery, one patient with a trisectectomy and two patients with atypical resection. Of the 13 liver lesions treated, five were local recurrences after radiofrequency ablation or atypical resection.

To objectify patient selection, a pre-interventional evaluation of sonographic accessibility was dispensed with.

\section{Sonographically-assisted CT-supported Brachytherapy}

The catheter placement was performed in an 80-row CT unit (Aquillion Prime, Canon Medical Systems, Neuss, Germany) with concomitant analgosedation of the patient with on-demand, intravenous administration of fentanyl and midazolam under pulse oximetry monitoring. The interstitial access to the target lesion was performed via an initial, image-guided puncture (if possible, sonographically-guided, otherwise performed CT-fluoroscopically) with an $18 \mathrm{Ga}$ coaxial needle and the subsequent change to $25 \mathrm{~cm}$-long 6F catheter sheaths (Terumo Radifocus ${ }^{\circledR}$ Introducer II, Terumo Europe, Leuven, Belgium) over a stiff guidewire (Amplatz SuperStiff TM, Boston Scientific, Marlborough, USA). A 6F irradiation catheter (afterloading catheter, Primed ${ }^{\circledR}$ Medizintechnik $\mathrm{GmbH}$, Halberstadt, Germany) was placed flush with the inner lumen of the sheath and the system was fixed with a skin suture.

If the lesions were small and round $(<4 \mathrm{~cm})$, an irradiation catheter was inserted into a central position. In the case of a larger or irregularly shaped lesion, multiple catheters were inserted to match the shape of the ablation zone (depending on the access path in a fanned or crossed arrangement).

Once the patient was brought into the radiotherapy site, treatment planning was carried out using a planning CT (Oncentra ${ }^{\circledR}$ Brachy, Elekta Instrument AB, Stockholm, Sweden) and single fraction irradiation with an iridium 192 source used in afterloading technique. After defining the gross tumor volume (GTV) based on the available image information, a safety margin of $5 \mathrm{~mm}$ was added for the computer-assisted generation of the clinical target volume (CTV). Due to the stable catheter position in the target volume, the CTV could then be equated with the final planning target volume (PTV). Depending on the tumor entity, a target dose of $15 \mathrm{~Gy}$ (renal cell carcinoma, hepatocellular carcinoma), 20 Gy (cholangiocarcinoma carcinoma) or 25 Gy (colorectal liver metastasis) was prescribed for CTV/PTV.

Within the study, CT fluoroscopy (120 kVp / 30 mAs, 0.5 s rotation time, $6 \mathrm{~mm}$ single-slice acquisition, image matrix $512 \times 512$ ) was replaced by laterally-positioned sonography using low-frequency convex $(1-5 \mathrm{MHz})$ and matrix $(1-6 \mathrm{MHz})$ ultrasound heads (EPIQ7, Philips Medical Systems, Amsterdam, The Netherlands) during the initial puncture and interim position monitoring as often as technically feasable (see $>$ Fig. 1 ). The free-hand punc- 
ture technique was used for eight lesions, while four lesions were punctured via a coaxial guide on the ultrasound head.

At the end of each procedure, contrast-enhanced computed tomography was performed as needed to perform radiotherapy planning.

Two specialists in radiology with 7 and 4 years experience in percutaneous interventions (at least 1000 and 300 documented percutaneous interventions, respectively) were responsible for the performance and assessment of the interventions.

\section{Study Design and Statistics}

Patient characteristics, the number of catheters per imaging modality, intervention and lesion, lesion parameters, and fluoroscopy times were tabulated. The image datasets were recorded for each intervention performed, and the visibility of the lesions by the intervention radiologists involved was assessed by consensus using a grading scale. In addition, the dose information (CTV, target dose, D100) of all lesions treated was collected from the treatment planning system.

The Society for Interventional Radiology (SIR) classification was used to evaluate major and minor complications [10].

The collected data were first descriptively evaluated in SPSS 24.0 (IBM $^{\circledR}$ SPSS $^{\circledR}$ Statistics, IBM Deutschland GmbH, Ehningen, Germany) with determination of mean and standard deviation as well as median and spread. Box plots were used to illustrate the data. If a comparison of statistical variables between CT and ultrasound imaging modalities was methodically feasible in the small patient population, this was done by the Mann-Whitney $U$ test for independent samples and the Wilcoxon signed-rank test.

\section{Results}

\section{Image-guided Catheter Positioning}

A total of 16 tumors with a mean diameter of $3.9 \pm 2.7 \mathrm{~cm}$ (min $1.5 \mathrm{~cm}$ to max. $12.9 \mathrm{~cm}$ ) were treated using $2.3 \pm 1.5$ irradiation catheters ( 1 to 5 catheters per lesion, 28 catheters in total).

Catheter positioning could be completely achieved under ultrasound guidance in 12 of 16 lesions and 23 of 28 catheters. In 4 tumors, the initial puncture had to be performed under CT fluoroscopy due to insufficient sonographic conditions. One liver lesion was directly beneath the diaphragm at a resection margin after trisectorectomy and another directly in the liver hilus. In 2 other tumors, sonographic visibility was not considered sufficient for an accurate puncture.

On the whole, all punctures and catheter placements of kidney tumors and liver tumors in the caudal segment row (segment $3 /$ $4 B / 5 / 6$ ) were successfully performed sonographically. In one patient with a lesion not visible in $C T$, sonographically-assisted puncture and catheter placement completely replaced CT fluoroscopy (see - Fig. 2). Here, only computed tomography with contrast agent application was necessary for radiation planning.

The planned target volume (CTV) dose was achieved in 14 of 16 lesions. In two cases the dose was reduced due to the proximity of the tumor treated to risk organs (gall bladder, maximum dose of 20 Gy, stomach / duodenum, maximum dose of 14 Gy).

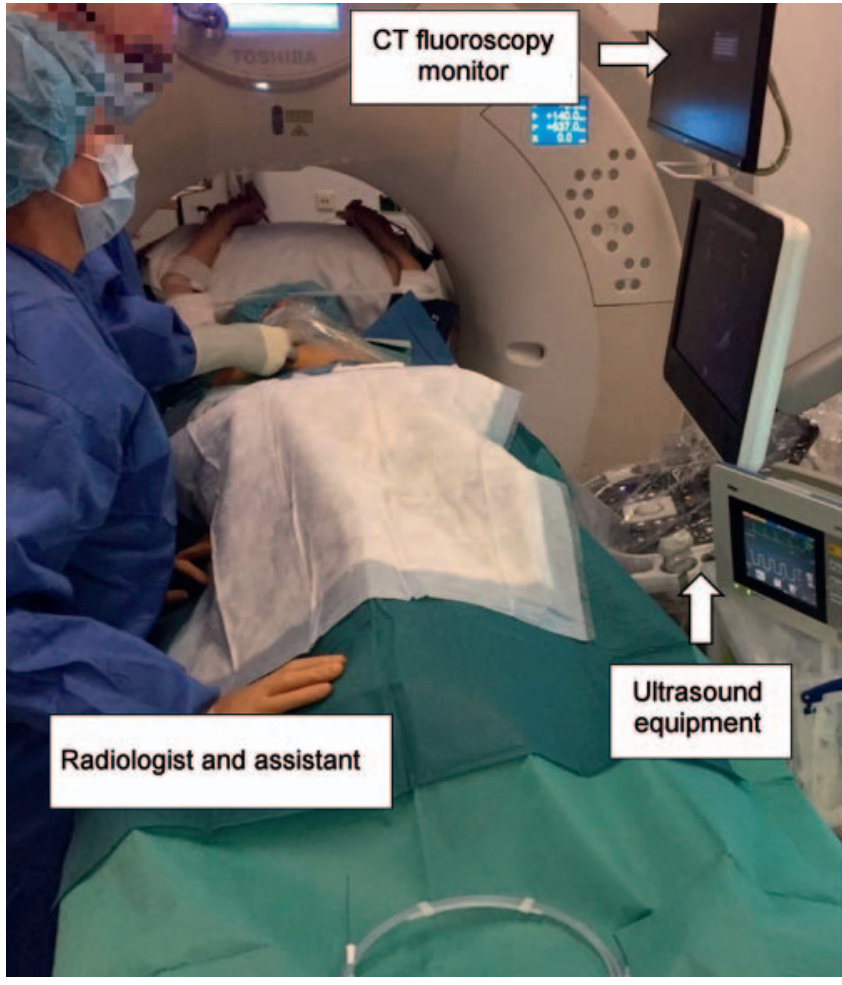

- Fig. 1 Arrangement in the CT room for ultrasound-assisted punctures.

\section{Fluoroscopy Time and Lesion Visibility}

In some cases of ultrasound-guided intervention, interim catheter placement controls were performed using CT fluoroscopy (120 $\mathrm{kVp}$ / $30 \mathrm{mAs}$, $0.5 \mathrm{~s}$ rotation time, $6 \mathrm{~mm}$ single-slice acquisition, image matrix $512 \times 512$ ). Mean fluoroscopy time for otherwise sonographically-guided procedures, however, was significantly shorter $(p=0.006$, see $>$ Fig. 3 ) at 14.5 s versus 105.5 s when CT fluoroscopy was used for the whole procedure.

The visibility of the target lesions was assessed based on the consensus of the two radiologists for both imaging modalities. In sonography, recognizability was rated as very good in 8 out of 16 lesions based on graded assessments; in CT fluoroscopy, this was only true for 2 lesions. Four or seven lesions were graded as good, two or five were considered satisfactory. In 2 tumors there was deficient detectability in the ultrasound (two cholangiocarcinomas) or CT (one hepatocellular carcinoma, one colorectal liver metastasis). Other grades were not issued in the low number of cases. Fig. 4 provides an overview of the assessment of lesion visibility. Statistically, there was no significant difference between the modalities ( $p=0.27$ ), although visibility was better in sonography compared to CT in a total of 6 lesions.

\section{Interstitial Tumor Ablation}

The tumor-enclosing dose during single fraction irradiation was set at 15 to $25 \mathrm{~Gy}$, depending on the tumor entity, and the mean target dose was $20.6 \pm 4.0 \mathrm{~Gy}$. With respect to the clinical target volume (CTV), the final dose distribution (D100) reached the target dose in 14 out of 16 cases, averaging 19.3 $\pm 4.8 \mathrm{~Gy}$. In two pa- 


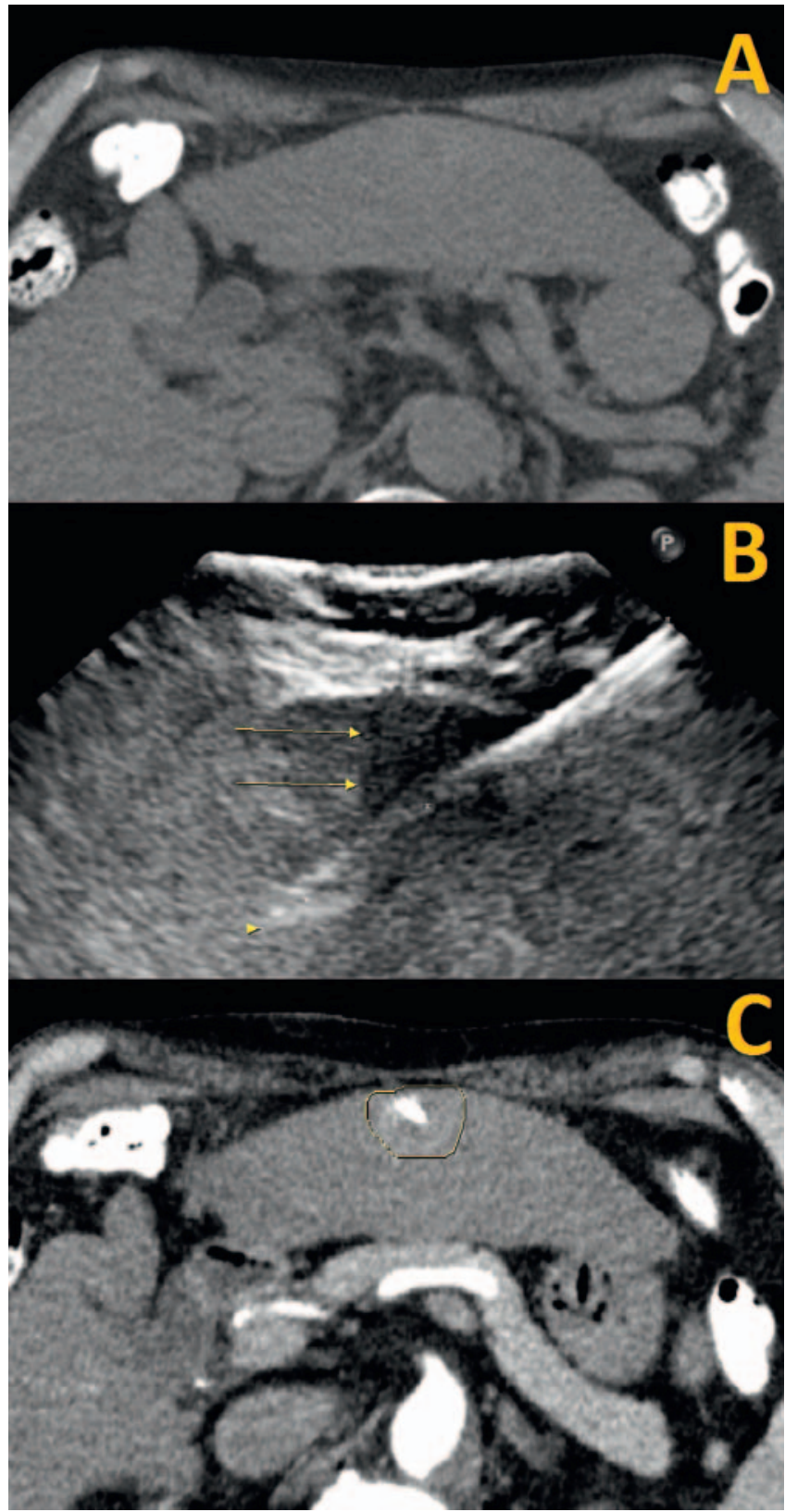

- Fig. 2 Interstitial brachytherapy of a subcapsular HCC in liver segment III. The lesion is barely visible in non-enhanced CT $\mathbf{A}$ although being detected in previous MRI. Sonography $\mathbf{B}$ demonstrates a good visualization of the HCC as a hypoechogenic mass (arrows) and is easily punctured (arrow head depicting the needle tip). Contrastenhanced $\mathrm{CT}$ in the arterial phase for irradiation planning $\mathbf{C}$ showing a central location of the catheter in the HCC (circle).

tients, the reason for the reduced dose was the proximity of the tumor to neighboring radiation-sensitive organs (stomach $n=1$, duodenum $n=1$ ).

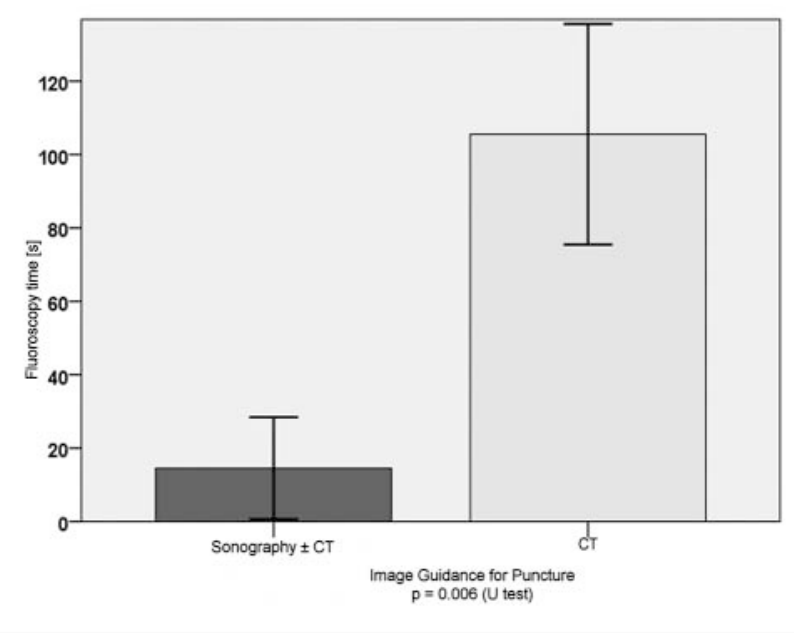

- Fig. 3 Mean fluoroscopy time ( \pm standard deviation) for ultrasound-assisted puncture (Sonography \pm CT) vs. CT puncture alone (CT) during catheter placement.

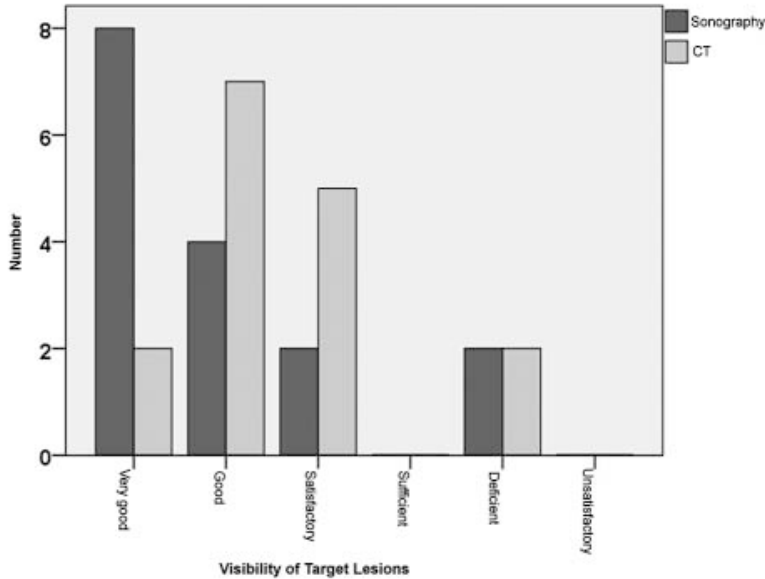

- Fig. 4 Visibility of the target lesion $(n=16)$ by imaging modality (sonography vs. CT).

\section{Complications}

After removal of the catheter sheaths, a sonographic or CT check was carried out after approximately two hours to rule out acute hemorrhaging in all cases.

At the 30-day follow-up, no major or minor complications were observed in the patient population after sonographically-assisted or direct CT fluoroscopic catheter placement.

\section{Discussion}

To the best of our knowledge, this feasibility study was the first to utilize sonography during image-guided interstitial HDR brachytherapy of hepatic and renal tumors as an image guidance modality for the initial puncture and catheter insertion. Previously only CT or MRI fluoroscopy were used [11, 12]. 
In an initial exploratory analysis, it was shown that a majority of catheter placements for CT-guided HDR brachytherapy can be performed with sonography equipment additionally positioned adjacent to the $\mathrm{CT}$ table. The kidneys as well as the caudal liver segments (3/4B/5/6) appeared to be particularly suitable as sonographicallyaccessible regions; in the previous patient cohort only a few lesions in one of cranial liver segments $(2 / 4 \mathrm{~A} / 7 / 8)$ were inaccessible. Here the results are in line with studies that, for example, have assessed the value of ultrasound and CT for radiofrequency ablation of hepatocellular carcinomas and which were able to document comparable results [9]. However, the benefits of CT fluoroscopy are also known when, similar to our cohort, certain regions of the abdomen are difficult for ultrasound to access $[13,14]$. However, many percutaneous procedures in interventional radiology still lack a comparative, randomized study between sonography and CT fluoroscopy.

The significance of the study is primarily limited by the small number of patients on whom the possibilities of the novel technique was observed. The goal should now be to use a larger number of cases to define the value of ultrasound-assisted catheter placement for a practical implementation in CT-guided HDR brachytherapy. A suitable comparison criterion appears to be the reduction of fluoroscopy time during $\mathrm{CT}$, which is proportional to the radiation exposure of the medical staff involved and could already be significantly reduced in the present study with the aid of ultrasound [15]. Similarly, the immediate availability of a second imaging modality improves the visibility and accessibility of certain lesions similar to the principle of CT / ultrasound image fusion [16]. Ultimately, this may result in improved positioning of the irradiation catheters or a reduction in the number needed for sufficient irradiation. For radiotherapy planning, however, CT imaging will continue to be required, and the procedure will therefore not be fully within the field of sonography.

\section{Summary}

Sonographically-assisted catheterization in interstitial HDR brachytherapy has the potential to reduce the use of CT fluoroscopy and therefore the radiation exposure of the interventional radiologist. The visibility of the target lesion in sonography is in some cases superior to CT fluoroscopy and allows accurate catheter placement even in previously operated patients.

The approaches gained from this study are intended to develop the concrete added value of the procedure in subsequent investigations on a larger group of patients.

\section{CENTRAL STATEMENTS / CLINICAL RELEVANCE}

- Ultrasound-assisted catheterization during CT-guided brachytherapy of abdominal tumors is technically feasible and safe.

- Ultrasound-based puncture can improve catheter placement.

- A significant reduction in fluoroscopy time can help reduce the radiation exposure of medical personnel.

\section{Conflict of Interest}

The authors declare that they have no conflict of interest.

\section{References}

[1] Collettini F, Singh A, Schnapauff D et al. Computed-tomography-guided high-dose-rate brachytherapy (CT-HDRBT) ablation of metastases adjacent to the liver hilum. Eur J Radiol 2013; 82: e509-e514

[2] Yang W, Yan K, Wu GX et al. Radiofrequency ablation of hepatocellular carcinoma in difficult locations: Strategies and long-term outcomes. World J Gastroenterol 2015; 21: 1554-1566

[3] Lin ZY, Li GL, Chen J et al. Effect of heat sink on the recurrence of small malignant hepatic tumors after radiofrequency ablation. J Cancer Res Ther 2016; 12: C153-C158

[4] Van den Begin R, Engels B, Gevaert T et al. Impact of inadequate respiratory motion management in SBRT for oligometastatic colorectal cancer. Radiother Oncol 2014; 113: 235-239

[5] Kloeckner R, dos SantosDP, Schneider J et al. Radiation exposure in CT-guided interventions. Eur J Radiol 2013; 82: 2253-2257

[6] Rogits B, Jungnickel K, Lowenthal D et al. Prospective Evaluation of the radiologist's hand dose in CT-guided interventions. Rofo 2013; 185: $1081-1088$

[7] Gianfelice D, Lepanto L, Perreault P et al. Effect of the learning process on procedure times and radiation exposure for $\mathrm{CT}$ fluoroscopy-guided percutaneous biopsy procedures. J Vasc Interv Radiol 2000; 11: 1217-1221

[8] Schaefer PJ, Schaefer FKW, Heller M et al. CT fluoroscopy-guided biopsy of small pulmonary and upper abdominal lesions: Efficacy with a modified breathing technique. J Vasc Interv Radiol 2007; 18: 1241 - 1248

[9] Wu J, Chen P, Xie YG et al. Comparison of the effectiveness and safety of ultrasound- and CT-guided percutaneous radiofrequency ablation of non-operation hepatocellular carcinoma. Pathol Oncol Res 2015; 21: $637-642$

[10] Sacks D, McClenny TE, Cardella JF et al. Society of Interventional Radiology clinical practice guidelines. J Vasc Interv Radiol 2003; 14: S199-S202

[11] Collettini F, Lutter A, Schnapauff D et al. Unresectable colorectal liver metastases: percutaneous ablation using $\mathrm{CT}$-guided high-dose-rate brachytherapy (CT-HDBRT). Rofo 2014; 186: 606-612

[12] Ricke J, Thormann M, Ludewig M et al. MR-guided liver tumor ablation employing open high-field 1. OT MRI for image-guided brachytherapy. Eur Radiol 2010; 20: 1985-1993

[13] Sheafor DH, Paulson EK, Kliewer MA et al. Comparison of sonographic and CT guidance techniques: does CT fluoroscopy decrease procedure time? Am J Roentgenol 2000; 174: 939-942

[14] Mukhtar KN, Mahmood SN, Umair SF. CT guided percutaneous renal biopsy versus ultrasound guided for obtaining adequate tissue. J Pak Med Assoc 2012; 62: 880-882

[15] Rathmann N, Haeusler U, Diezler P et al. Evaluation of radiation exposure of medical staff during CT-guided interventions. J Am Coll Radiol 2015; 12: $82-89$

[16] Lee MW, Rhim H, Cha DI et al. Planning US for percutaneous radiofrequency ablation of small hepatocellular carcinomas $(1-3 \mathrm{~cm})$ : value of fusion imaging with conventional US and CT/MR images. J Vasc Interv Radiol 2013; 24: $958-965$ 
